\title{
More Controversy than Ever - Challenges and Promises Towards Personalized Treatment of Gastric Cancer
}

\author{
Theodore Liakakos, MD, and Dimitrios H. Roukos, MD \\ Department of Surgery, Ioannina University School of Medicine, Ioannina University, GR-45110, Ioannina, 45, Greece
}

Clinical decisions for the treatment of localized gastric cancer have become much more sophisticated and complicated than ever. Two recent large-scale trials published in NEJM for East Asian ${ }^{1}$ and Western patients ${ }^{2}$ strongly support the routine use of adjuvant chemotherapy. However, differences in design, extent of surgery, kind of chemotherapy timing of administration, and survival results in the two trials as well as potential differences in genetic background of Asian and Western gastric caner patients raise critical questions and grow confusion and uncertainty. Which is the optimum update treatment for Western patients?

Is the Japanese model with standardized D2 surgery followed by one year S-1 chemotherapy applicable in the West and can it produce similar excellent results or should treatment decisions be based on Western patient's data from the UK MAGIC trial ${ }^{2}$ and the USA INT-0116 trial? ${ }^{3}$ This editorial approaches this critical question towards a live-saving decision. Emphasis is given to current advances in network biology, ${ }^{4}$ cancer genome and functional studies $^{5-10}$ as well as a current comprehensive benchto-bedside genomic-based protocol for biomarkersbased personalized treatment of gastric cancer. ${ }^{11}$

The landmark ACTS-GC Japanese study demonstrated excellent survival results with primary standardized D2 surgery followed by S-1 chemotherapy

Published online January 23, 2008.

Address correspondence and reprint requests to: Dimitrios $\mathrm{H}$. Roukos, MD; E-mail: droukos@cc.uoi.gr

Published by Springer Science+Business Media, LLC @ 2008 The Society of Surgical Oncology, Inc. for advanced stages II and III. ${ }^{1}$ Most patients $(89 \%)$ had node-positive disease; these advanced tumor stages are associated with poor prognosis in the West. ${ }^{12}$ Despite this advanced disease, the overall 3year survival rate was $70 \%$ after D2 surgery alone and $80 \%$ in the S-1 chemotherapy group. The hazard ratio for death in the S-1 group, as compared with the surgery-only group, was 0.68 (95\% CI, $0.52-0.87$; $p=0.003$ ). Because of this significant survival difference the trial was stopped.

Local control is now increasingly recognized to have a crucial role in the treatment strategy not only of gastric cancer ${ }^{13-15}$ but also for many other solid tumor including breast cancer despite the use of adjuvant treatment. ${ }^{16,17}$ Appropriate surgery alone or surgery plus radiotherapy for local control not only reduce local recurrence but also improve overall survival. ${ }^{17}$ This principle could be achieved by a perfectly conducted standardized D2 surgery. The 109 centers located throughout Japan were selected from among hospitals performing at least 100 operations annually for gastric cancer. This highquality D2 surgery had resulted in a local relapse rate of only $2.8 \%$ and a nodal relapse rate of $8.7 \%$. Adjuvant S-1chemotherapy significantly reduced all types of recurrence (peritoneal, local, nodal) but questions arise form the finding that systemic treatment did not improve hematogenous relapse $(p=0.35)$.

The ACTS-GC study provides some important messages but also raises several questions. First, can the Japanese excellent results be reproduced in Western patients? There is long-term discussion on whether there are differences between Japanese and 
Western gastric cancer and patients. Sakuramoto and colleagues believe that these results can be achieved in the West if a standardized, Japanese-style D2 surgery is performed, because there is no genetic difference between gastric cancers in Japan and Europe. Although this may be correct, for definitive conclusions much more in-depth research work is needed considering gastric cancer genome and interactions between environmental and genetic factors in Japan and Europe or the USA. If all these genetic alterations and environmental variables in carcinogenesis and metastasis are similar, then indeed the Japanese results can be reproduced in the West. It should be noted, however, that survival results for gastric cancer even with adequate D2 surgery in highly specialized Western institutions, ${ }^{18-20}$ are much lower than those reported from Japan. ${ }^{1,13}$

Second, the Japanese study has demonstrated that a substantial fraction of stage II/III patients had a localized nature of disease. Indeed, the estimated actuarial 5-year relapse-free survival was approximately $50 \%$ in the surgery-only group. How can these patients be identified to spare chemotherapy toxicity? Third, the addition of chemotherapy after perfect local and nodal control could increase the survival rate by $10 \%$. How can this small survival benefit be further improved? These two questions are discussed below as they can be approached considering current and future research directions towards the development of both novel biomarkers and targeted agents.

Preoperative and postoperative (perioperative) chemotherapy with epirubicin, cisplatin, and fluorouracil was associated with a 5-year overall survival benefit of $13 \%$ (from $23 \%$ to $36 \%$ ) for stage II or III operable gastric cancer patients in the MAGIC trial conducted mainly in the UK. ${ }^{2}$ In the USA INT-0116 trial, ${ }^{3}$ postoperative chemoradiotherapy improved the 3 -year overall survival from $41 \%$ to $50 \%$. Both trials have been criticized for inadequate surgery (D0 or D1). Thus, the approximately $30 \%$ survival difference between the Japanese trial ${ }^{1}$ and Western trials $^{2,3}$ could likely be explained by the fact that chemotherapy or chemoradiotherapy simply compensated mainly surgical failure for local and nodal control. ${ }^{13,21,22}$ Another reason, beyond the potential differences in body mass index that encourages an "easy" D2 surgery for Japanese patients, cancer genome and environment, is also the inclusion of patients with gastroesophageal junction tumors, which are associated with poorer survival, in the Western trials ${ }^{2,3}$ while such patients were excluded from the Japanese study.

\section{PERSONALIZED TREATMENT}

Worldwide, despite improvements, estimated cure rates for patients with stages IIIA and IIIB remain poor. Given the short mean follow-up of 3 years in the ACTS-GC study and the fact that a subset of patients, approximately $20 \%$, recur after the three first postoperative years, ${ }^{23}$ a cure rate of approximately $35 \%$ with surgery only and $45 \%$ by adding S1 chemotherapy to D2 surgery could be estimated. These rates are currently the best achieved worldwide; those reported from the West are lower despite D2 surgery and adjuvant treatment. ${ }^{18-20,24,25}$ These clinical data strongly indicate the urgent need to develop new therapeutic strategies. .

How can long-term survival results be improved? Despite intensive efforts over recent decades, singlegene-based traditional research has produced little clinical success. No robust prognostic or predictive marker has been validated for clinical use despite multiple reports and hundreds of proposals. ${ }^{26} \mathrm{An}$ isolated success of traditional research towards personalized medicine for various cancer types ${ }^{27,28}$ including gastric cancer is the prevention and treatment of hereditary diffuse gastric cancer (HDGC). ${ }^{29}$ Individuals who are carriers of germ-line mutations in the $C D H 1$ (E-cadherin) gene face a very high lifetime risk of gastric cancer $(60-80 \%) .{ }^{30}$ This very small subpopulation can be identified from the very large general population on the basis of family history criteria and genetic testing. ${ }^{30}$ Tailoring a riskreducing prophylactic gastrectomy for these very high-risk individuals can save their lives. ${ }^{31}$ Women with $\mathrm{CDH1}$ mutations also have a high risk, approximately $40 \%$, of breast cancer. ${ }^{32}$ For these carriers, as well as for women with germ-line $B R C A 1 /$ 2 mutations, ${ }^{33}$ prophylactic bilateral mastectomy ${ }^{34,35}$ or close surveillance ${ }^{36}$ could be considered. ${ }^{37}$

But the management of sporadic cancer is much more complicated because multiple genes and oncogenic pathways are involved in gastric carcinogenesis. ${ }^{6}$ Obviously, single-gene-based traditional research cannot reveal the global signaling pathways network. Gastric cancer is a biologically heterogeneous disease with various molecular tumor subsets that likely respond distinctly differently and require new therapeutics for complete response. In the postgenomic era of network cancer biology, ${ }^{4}$ and with the advent of the new high-throughput technologies language, methodologies and research directions should be altered for a faster clinical success. ${ }^{5-10}$

Currently, a comprehensive bench-to-bedside genomic-based model for personalized treatment of 
Pretreatment clinical staging: Patients with stage II and III gastric cancer

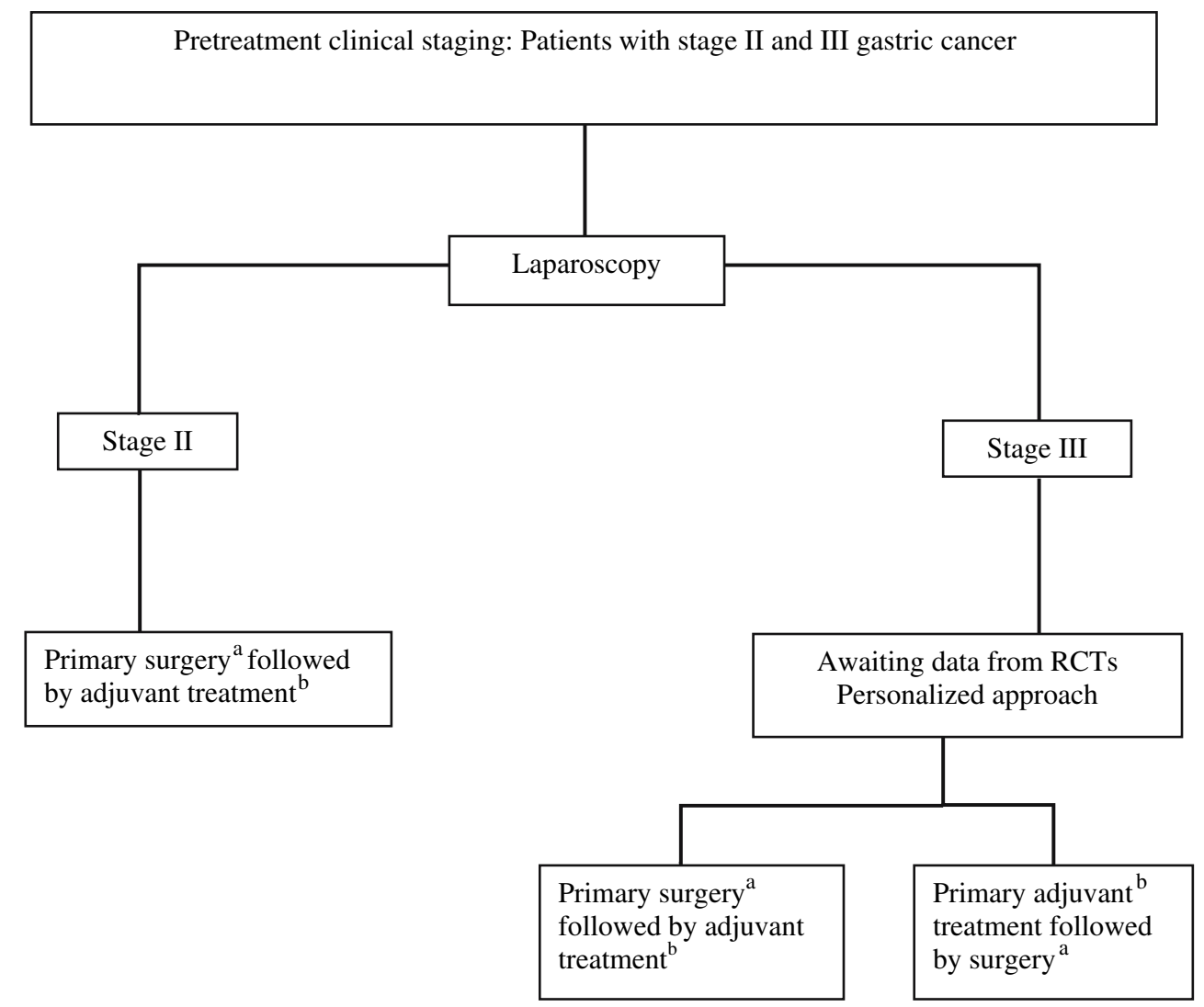

FIG. 1. Flow chart for decision-making regarding adjuvant treatment for resectable gastric cancer. ${ }^{\text {a } D 2}$ surgery if surgeon's experience ensures a safe and effective procedure. If a standardized D2 surgery is not feasible, D1 surgery with chemoradiotherapy ${ }^{3}$ or preoperative chemotherapy ${ }^{2}$ can be considered. ${ }^{b}$ Adjuvant treatment: Cisplatin-based chemotherapy for Western patients, S-1 chemotherapy for East Asian patients. Addition of targeted agents to empirical chemotherapy only in randomized trials. RCTs: randomized controlled trials.

gastric cancer has been developed and proposed by Roukos. ${ }^{11}$ This protocol has been focused on how to overcome current treatment challenges and biases associated with high-throughput analyses. As reported above, the vast majority of patients received an inadequate adjuvant treatment either because they do not need adjuvant systemic treatment, they have a localized nature of disease, or they do not respond to currently used cytotoxic chemotherapy. Focusing on these clinical needs, cancer genome and functional strategies are integrated to reveal the precise role of key genes and interacting pathways. ${ }^{4-10}$ The proposed model can lead to the development of class II prognostic and class III predictive biomarkers. ${ }^{38}$ Profiling involved gastric cancer key genes, driver mutations, and interacting pathways will improve our understanding on signaling pathways network. Insights into the presence or absence of small cells subpopulations like cells with high mutation rates or cancer stem-like cells in individual tumors will clarify resistance and sensitivity to cytotoxic and targeted agents. Taken together, the development of both novel biomarkers and targeted agents can be achieved. Prognostic gene signatures might classify patients into low and high metastatic risk. Low-risk patients can successfully be treated by R0 surgery alone, sparing unnecessary chemotherapy toxicity. Among patients at high risk of metastatic relapse, predictive gene signatures can be developed comparing tissue-bank samples from responding and nonresponding patients. The fact that the protocol has considered, from the very initiating stage of design, the current robust clinicopathological factors including the Lauren classification into intestinaltype and diffuse-type gastric cancer, enables the clinical prospective validation of new biomarkers. ${ }^{11}$

\section{CURRENT CLINICAL PRACTICE}

Although cancer genome and functional studies provide great promise for the future, patients and 
clinicians are currently face hard decisions: preoperative or postoperative chemotherapy combined with D2 surgery or D1 plus radiotherapy? Considering the preclinical and clinical evidence, a step-by-step treatment-guided algorithm for Western clinical practice is provided (Fig. 1). Laparoscopy is clinically useful to confirm by biopsy or exclude distant metastasis (peritoneum, liver, distant abdominal enlarged lymph nodes) and serosa status (serosa negative or positive) for more accurate staging than clinical staging with endoscopic ultrasonography, computer tomography or positron emission tomography (PET). Analysis of data from randomized clinical trials $(\mathrm{RCTs})^{1-3,39}$ and descriptive studies show that, first, survival rates for stage II patients are relatively good not only in Japan ${ }^{1,13}$ but also in the West ${ }^{18,19,40}$ with appropriate surgery followed by adjuvant treatment. Because preoperative chemotherapy harms some of these patients ${ }^{22}$ primary D2 surgery without delay, converting laparoscopy into an open D2 gastrectomy, now appears to be the preferred approach. Laparoscopic-assisted D2 gastrectomy provides encouraging data but feasibility, safety, and efficacy can be ensured at the present time only in a few highly specialized institutions. ${ }^{41}$ Second, because postoperative S-1 chemotherapy for stages IIIA and IIIB did not significantly improve survival in the subgroup analysis of the ACTS-GC study, ${ }^{1}$ preoperative cisplatin-based chemotherapy for downstaging and potential early elimination of circulated cancer cells ${ }^{2}$ could be considered, although no subgroup analysis from the MAGIC trial is available.

\section{CONCLUSIONS}

Gastric cancer still remains a major health problem. Despite improvements in local control and empirical chemotherapy, prognosis particularly for stage III patients remains poor worldwide. New therapeutic strategies are needed.

In the postgenomic era of network cancer biology, clinical practice could be altered when the catalogue of gastric cancer genes has been completed and new high-throughput analyses reveal the whole genome function, including the understanding of noncoding DNA regions. In the meantime, microarray-based and proteomics data profiling genes, mutations, and interacting oncogenic signaling pathways may lead to the development and validation of both biologically targeted agents and prognostic class II and predictive class III biomarkers. Tailoring the best preoperative or postoperative combination of empirical cytotoxic and targeted agents distinctly different in various subsets of individual tumors on the basis of which genes are involved and which pathways are deregulated in a give patient, then indeed personal cancer genome and personalized oncology will revolutionize clinical outcomes not only of gastric cancer but also most solid cancers. ${ }^{11}$

\section{REFERENCES}

1. Sakuramoto S, Sasako M, Yamaguchi T, et al. Adjuvant chemotherapy for gastric cancer with S-1, an oral fluoropyrimidine. $N$ Engl J Med 2007; 357:1810-20.

2. Cunningham D, Allum WH, Stenning SP, et al. Perioperative chemotherapy versus surgery alone for resectable gastroesophageal cancer. $N$ Engl J Med 2006; 355:11-20.

3. Macdonald JS, Smalley SR, Benedetti J, et al. Chemoradiotherapy after surgery compared with surgery alone for adenocarcinoma of the stomach or gastroesophageal junction. $N$ Engl J Med 2001; 345:725-30.

4. Friedman A, Perrimon N. Genetic screening for signal transduction in the era of network biology. Cell 2007; 128:225-31.

5. Bild AH, Potti A, Nevins JR. Linking oncogenic pathways with therapeutic opportunities. Nature Rev Canc 2006; 6:73541.

6. Greenman C, Stephens P, Smith R, et al. Patterns of somatic mutation in human cancer genomes. Nature 2007; 446:153-8.

7. Thomas RK, Baker AC, Debiasi RM, et al. High-throughput oncogene mutation profiling in human cancer. Nat Genet 2007; 39:347-51

8. Wood LD, Parsons DW, Jones S. The genomic landscapes of human breast and colorectal cancers. Science. 2007; 318:110813.

9. ENCODE Project Consortium Birney E, Stamatoyannopoulos JA, Dutta A, et al. Identification and analysis of functional elements in $1 \%$ of the human genome by the ENCODE pilot project. Nature 2007; 447:799-816.

10. Hanahan D, Weinberg RA. The hallmarks of cancer. Cell 2000; 100:57-70.

11. Roukos DH. Innovative genomic-based model for personalized treatment of gastric cancer - integrating current standards and new technologies. Expert Rev Mol Diagn 2008; 8:29-39.

12. Roukos DH, Kappas AM. Perspectives in the treatment of gastric cancer. Nat Clin Pract Oncol 2005; 2:98-107.

13. Sano T. Tailoring treatments for curable gastric cancer. $\mathrm{Br} J$ Surg 2007; 94:263-4.

14. Roukos DH. Extended lymphadenectomy in gastric cancer: when, for whom and why. Ann R Coll Surg Engl 1998; 80:1624.

15. Roukos DH. Extended (D2) lymph node dissection for gastric cancer: do patients benefit?. Ann Surg Oncol 2000; 7:253-5.

16. Clarke M, Collins R, Darby S, et al. Effects of radiotherapy and of differences in the extent of surgery for early breast cancer on local recurrence and 15-year survival: an overview of the randomized trials. Lancet 2005; 366:2087-106.

17. Punglia RS, Morrow M, Winer EP, et al. Local therapy and survival in breast cancer. $N$ Engl J Med 2007; 356: 2399-405.

18. Siewert JR, Bottcher K, Stein HJ, et al. Relevant prognostic factors in gastric cancer: ten-year results of the German Gastric Cancer Study. Ann Surg 1998; 228:449-61.

19. Brennan MF. Current status of surgery for gastric cancer: a review. Gastric Cancer 2005; 8:64-70.

20. Patel PR, Mansfield PF, Crane $\mathrm{CH}$, et al. Clinical stage after preoperative chemoradiation is a better predictor of patient 
outcome than the baseline stage for localized gastric cancer. Cancer 2007; 110:989-95.

21. Roukos DH. Adjuvant chemoradiotherapy in gastric cancer: wave goodbye to extensive surgery?. Ann Surg Oncol 2002; 9:220-1.

22. Briasoulis E, Fatouros M, Roukos DH. Level I evidence in support of perioperative chemotherapy for operable gastric cancer: sufficient for wide clinical use?. Ann Surg Oncol 2007; 14:2691-5.

23. Roukos DH, Lorenz M, Karakostas K, et al. Pathological serosa and node-based classification accurately predicts gastric cancer recurrence risk and outcome, and determines potential and limitation of a Japanese-style extensive surgery for Western patients: a prospective with quality control 10-year followup study. Br J Cancer 2000; 84:1602-9.

24. Kappas AM, Fatouros M, Roukos DH. Is it time to change surgical strategy for gastric cancer in the United States?. Ann Surg Oncol 2004; 11:727-30.

25. Roukos DH, Kappas AM. Targeting the optimal extent of lymph node dissection for gastric cancer. J Surg Oncol 2002; 81:59-62.

26. Briasoulis E, Liakakos T, Dova L, et al. Selecting a specific pre- or postoperative adjuvant therapy for individual patients with operable gastric cancer. Expert Rev Anticancer Ther 2006; 6:931-9.

27. Roukos DH, Murray S, Briasoulis E. Molecular genetic tools shape a roadmap towards a more accurate prognostic prediction and personalized management of cancer. Cancer Biol Ther 2007; 6:308-12.

28. Roukos DH. HER2 and response to paclitaxel in node-positive breast cancer. N Engl J Med 2008; 358:197, authors reply 198.

29. Norton JA, Ham CM, Van Dam J, et al. CDH1 truncating mutations in the E-cadherin gene: an indication for total gastrectomy to treat hereditary diffuse gastric cancer. Ann Surg 2007; 245:873-9.

30. Barber M, Fitzgerald RC, Caldas C. Familial gastric cancer aetiology and pathogenesis. Best Pract Res Clin Gastroenterol 2006; 20:721-34.
31. Roukos DH, Kappas AM, Tsianos E. Role of surgery in the prophylaxis of hereditary cancer syndromes. Ann Surg Oncol 2002; 9:607-9.

32. Chung DC, Yoon SS, Lauwers GY, Patel D. Case records of the Massachusetts General Hospital. Case 22-2007. A woman with a family history of gastric and breast cancer. $N$ Engl $J$ Med 2007; 357:283-91.

33. Roukos DH. Prognosis of breast cancer in carriers of BRCA1 and BRCA2 mutations. $N$ Engl $J$ Med 2007; 357:1555-6; author reply 1556.

34. Roukos DH, Agnanti NJ, Paraskevaidis E, et al. Approaching the dilemma between prophylactic bilateral mastectomy or oophorectomy for breast and ovarian cancer prevention in carriers of BRCA1 or BRCA2 mutations. Ann Surg Oncol 2002; 9:941-3.

35. Fatouros M, Baltoyiannis G, Roukos DH. The predominant role of surgery in the prevention and new trends in the surgical treatment of women with BRCA1/2 mutations. Ann Surg Oncol 2007 Oct 17; [Epub ahead of print]; DOI: 10.1245/s10434-007-9612-4.

36. Agnantis NJ, Paraskevaidis E, Roukos D. Preventing breast, ovarian cancer in BRCA carriers: rational of prophylactic surgery and promises of surveillance. Ann Surg Oncol 2004; 11:1030-4.

37. Roukos DH, Briasoulis E. Individualized preventive and therapeutic management of hereditary breast ovarian cancer. Nat Clin Pract Oncol 2007; 4:578-90.

38. Gewin V. Missing the mark. Nature 2007; 449:770-1.

39. Wu CW, Hsiung SA, Lo, et al. Nodal dissection for patients with gastric cancer: a randomised trial. Lancet Oncol 2006; 7:309-15.

40. Roukos DH. Early-stage gastric cancer: a highly treatable disease. Ann Surg Oncol 2004; 11:127-9.

41. Lee JH, Kim YW, Ryu KW, A phase-II clinical trial of laparoscopy-assisted distal gastrectomy with D2 lymph node dissection for gastric cancer patients. Ann Surg Oncol 2007 Aug 20; [Epub ahead of print]. 